\title{
Analyse de couches d'oxydation anodique d'acier inoxydable pour la conversion photothermique de l'énergie solaire
}

\author{
Lucien Ariès $\left({ }^{1}\right)$, Renée Calsou $\left({ }^{2}\right)$, Jean Antoine Florès $\left({ }^{1}\right)$ et Jean Pierre Traverse $\left({ }^{1}\right)$ \\ (1) Laboratoire de Recherche sur l'Énergie, Université Paul Sabatier, 31062 Toulouse Cedex, France \\ $\left({ }^{2}\right)$ Laboratoire de Microscopie et Structure des matériaux Université Paul Sabatier, 31062 Toulouse \\ Cedex, France
}

(Received May 9, 1990; accepted May 24, 1991)

\begin{abstract}
Résumé. - Dans une publication précédente, les modifications des propriétés provoquées par des traitements thermiques à l'air ont été présentées pour des couches sélectives préparées par traitement chimique. On procède à une étude analogue pour des couches préparées par oxydation anodique. La composition chimique, l'état de cristallisation, les propriétés optiques et la stabilité thermique sont analogues sous réserve que l'oxydation anodique soit effectuée à un potentiel proche du potentiel de corrosion naturelle. Les modifications de l'état d'oxydation et de la cristallinité obtenues à un potentiel plus élevé ne permettent pas d'améliorer la sélectivité optique ni la stabilité thermique.
\end{abstract}

\begin{abstract}
In a previous paper, modifications of characteristics induced by heat treatment in air are presented for selective coatings prepared by chemical treatment. Similar study is extended to coating prepared by anodic oxidation. Chemical composition, crystallisation state, optical properties and thermal stability are similar, provided that the anodic oxidation is carried out at a potential closed to natural corrosion potential. Enhanced oxidation and modified crystallinity obtained at higher potential favour neither optical selectivity nor thermal stability.
\end{abstract}

\section{Introduction}

Les couches de conversion d'aciers inoxydables que nous élaborons selon des procédés originaux [1,2] présentent un facteur d'absorption solaire très élevé $\left(a_{\mathrm{s}} \geq 0,95\right)$ et un faible facteur d'émission thermique $\left(\epsilon_{\curvearrowright} \leq 0,20\right)$ : ce sont des couches sélectives. Ces revêtements sont actuellement utilisés pour la fabrication industrielle de capteurs solaires plans.

Ces couches sont élaborées par conversion chimique superficielle d'aciers inoxydables en milieu acide et en présence d'espèces à base de chalcogène et notamment de sulfures ou de thiosulfates. Leurs caractéristiques dépendent de la nature (composition chimique, structure...) de l'acier inoxydable de départ ainsi que du mode et des conditions de traitement (température et composition du bain, durée de traitement...) [3,4].

Le traitement peut être effectué par voie chimique. Nous avons montré les caractéristiques remarquables de couches de conversion d'acier ferritique élaborées selon ce mode de traitement 
[5,6]. Elle présentent une excellente résistance à la dégradation thermique à l'air jusqu'à $450^{\circ} \mathrm{C}$. Utilisées comme absorbeurs de capteurs plans ; elles résistent à la dégradation thermique susceptible d'intervenir à la température de stagnation (de l'ordre de $200^{\circ} \mathrm{C}$ ); elles sont également adaptées pour les capteurs (sous vide ou utilisant une certaine concentration de l'énergie solaire pour lesquels les températures peuvent atteindre $400^{\circ} \mathrm{C}$ ).

Des couches sélectives peuvent être aussi obtenues par oxydation anodique. Cette méthode offre la possibilité de jouer sur la valeur du potentiel d'électrode de la surface à traiter. On peut ainsi espérer mieux contrôler la vitesse de croissance du revêtement, son épaisseur et sa composition. Ce procédé présente également l'avantage d'élargir la gamme des aciers inoxydables susceptibles de conduire à des couches de conversion ayant les caractéristiques requises pour un emploi donné (stabilité thermique, résistance à la corrosion...). Cependant ce procédé est plus couteux que le précédent.

Notre objectif est d'analyser l'influence du potentiel d'élaboration sur les caractéristiques de couches sélectives obtenues par oxydation anodique. En se référant à des couches analogues obtenues par voie chimique nous avons établi à l'état initial et après traitement thermique dans l'air jusqu'à $800^{\circ} \mathrm{C}$, les caractéristiques suivantes : propriétés optiques, composition chimique, état de cristallisation, épaisseur.

\section{Méthodes et conditions expérimentales.}

2.1 CARACTÉRISTIQUES DU SUBSTRAT. - Les couches sélectives étudiées sont préparées à partir d'acier inoxydable Z6 CNb17 dont la composition est donnée dans le tableau I.

Tableau I. - Composition massique de l'acier Z6 CNb17.

[Composition (weight percent) of stainless steel Z6 CNb17.]

\begin{tabular}{|lcccccccccc|}
\hline Elément & $\mathrm{C}$ & $\mathrm{Cr}$ & $\mathrm{Ni}$ & $\mathrm{Mo}$ & $\mathrm{Cu}$ & $\mathrm{Si}$ & $\mathrm{S}$ & $\mathrm{Ti}$ & $\mathrm{Nb}$ & $\mathrm{Fe}$ \\
$\begin{array}{l}\% \text { en } \\
\text { masse }\end{array}$ & 0,042 & 16,90 & 0,21 & 0,02 & 0,035 & 0,38 & 0,01 & 0,01 & 0,575 & 81,82 \\
\hline
\end{tabular}

Les échantillons se présentent sous la forme de tôle "recuit brillant" de quelques décimètres carrés et ne subissent pas de préparation mécanique. Elles sont de structure ferritique comme le montre la micrographie figure 1a. Nous avons effectué une analyse RHEED d'un échantillon traité préalablement par un léger polissage électrolytique pour supprimer la couche native de passivation. L'indexation du spectre (Fig.1b) confirme la structure ferritique de fer faiblement substitué en chrome, et montre la présence de carbonitrure de niobium dû au traitement industriel de l'alliage.

2.2 PRÉPARATION DES COUCHES. - Pour effectuer l'étude comparative des couches sélectives obtenues par voie chimique et par voie électrochimique, nous avons élaboré les couches dans les mêmes conditions de température et de composition de bain. Il s'agit des conditions d'élaboration optimales par rapport à la stabilité thermique déjà établies pour des couches de conversion par voie chimique [6].

Les conditions sont les suivantes :

- teneur en acide sulfurique $\mathrm{H}_{2} \mathrm{SO}_{4}: 15 \%$ en volume

- teneur en thiosulfate de sodium $\mathrm{Na}_{2} \mathrm{~S}_{2} \mathrm{O}_{3} 5 \mathrm{H}_{2} \mathrm{O}: 2 \times 10^{-3} \mathrm{~mol}^{-1}$ 

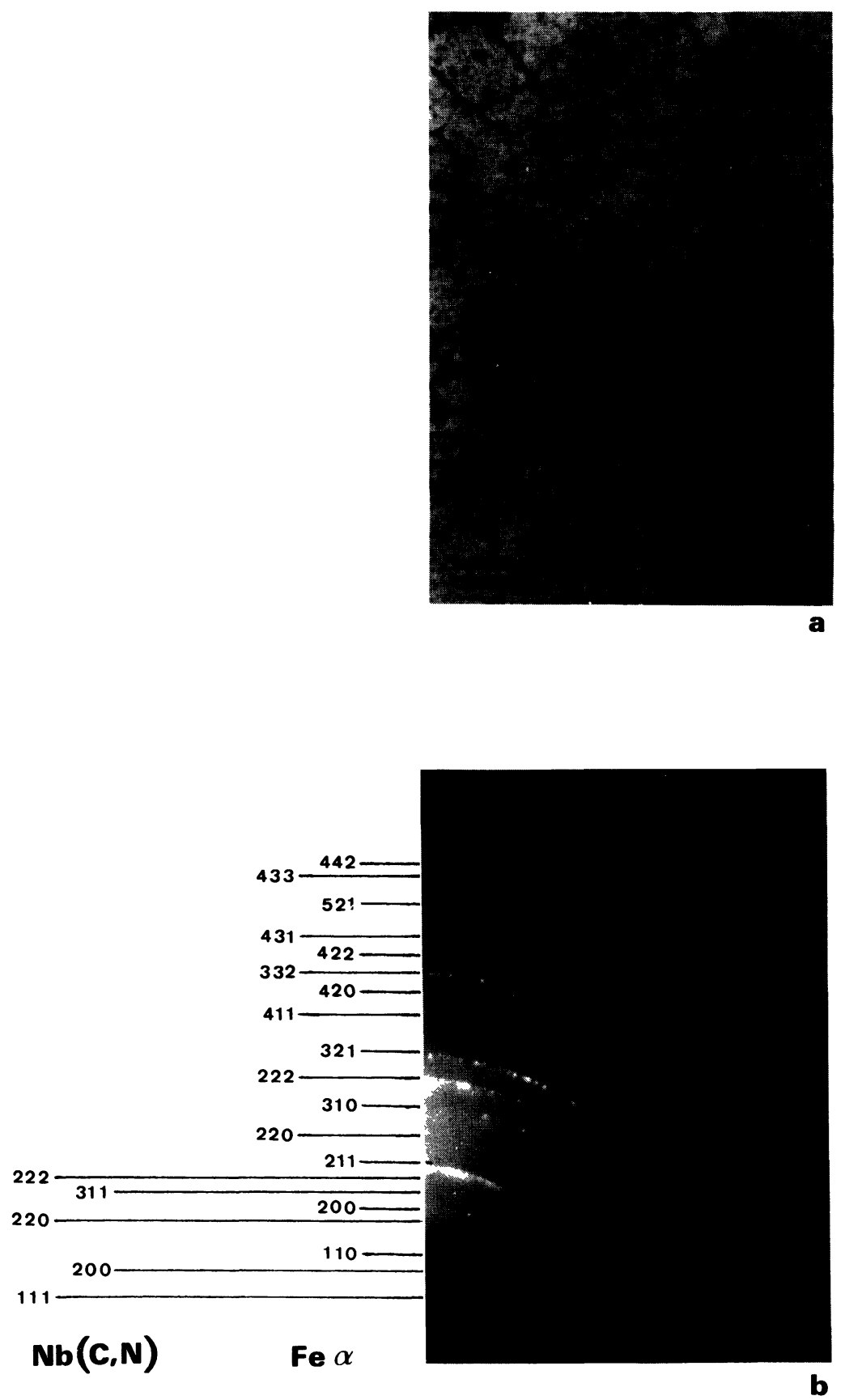

Fig. 1. - a) Micrographie optique de la structure de l'acier inoxydable ferritique Z8 CNb 17 . b) Spectre RHEED du substrat, l'échantillon a été soumis à un polissage-attaque électrolytique préalable. Polissage : tension : 40 volts, durée : 20 secondes. Attaque : tension : 15 volts, durée : 20 secondes.
[a) Optical micrography of the structure of stainless steel Z8CNb 17. b) Electron diffraction pattern of substrate of sample previously electrolytically etched.] 
- teneur en alcool propargylique $\mathrm{C}_{3} \mathrm{H}_{4} \mathrm{O}: 0,12$ mol. $^{-1}$

- température du bain $45^{\circ} \mathrm{C}$

La durée de traitement qui est de 5 minutes dans le cas du traitement chimique varie avec le potentiel de travail, et correspond, dans chaque cas, à l'obtention d'une sélectivité spectrale élevée.

Le traitement électrochimique est réalisé à potentiel d'electrode contrôlé et maintenu constant. Le montage comprend un potentiostat électronique et 3 électrodes. Les potentiels sont mesurés par rapport à une électrode au calomel saturé (ECS) et la contre électrode est une grille de platine de grande surface.

Après traitement, les échantillons sont rincés à l'eau déminéralisée puis séchés à l'air dans un four à $90^{\circ} \mathrm{C}$ pendant 5 minutes.

Le domaine de potentiel de traitement correspond au domaine d'activité de l'acier dans le bain d'élaboration (Fig. 2). Le potentiel d'abandon de l'acier dans ce bain est égal à $-460 \mathrm{mV}$ et le potentiel de passivation à $-180 \mathrm{mV}$. Pour chaque potentiel nous avons recherché la durée optimale de traitement qui conduit à des couches présentant la meilleure sélectivité spectrale. Cette durée d'élaboration diminue losque la densité de courant anodique augmente : elle est de l'ordre de quelques minutes au voisinage du potentiel de corrosion naturelle et de quelques dizaines de secondes au voisinage du potentiel de passivation.

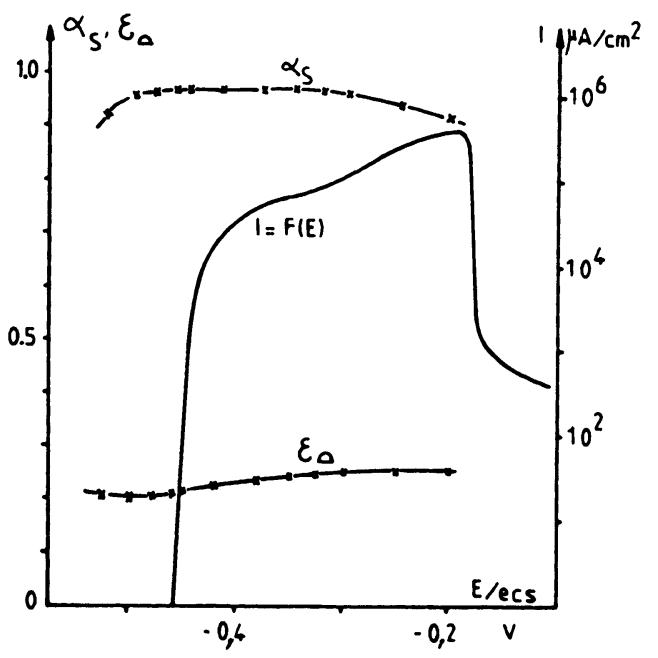

Fig. 2. - Variation des propriétés optiques des surfaces sélectives en fonction du potentiel et courbe de polarisation de l'acier dans le bain d'élaboration.

[Variation of optical properties of selective surfaces against potential and polarization curve of steel in treatment bath.]

2.3 TRAITEMENTS THERMIQUES. - Les traitements thermiques sont effectués à l'air à pression atmosphérique en système ouvert en utilisant des fours ouverts à l'atmosphère ambiante. La durée de maintien en température est de quinze jours ( 384 h) [6] ce qui correspond à une simulation des conditions d'utilisation. 
2.4 MESURE DES PROPRIÉTÉS OPTIQUES. - Le facteur d'absorption total, au voisinage de la normale, du rayonnement solaire, que nous appelons facteur d'absorption solaire $a_{s}$ est mesuré à l'aide d'un réflectomètre (Elan informatique EL 510). Ce facteur représente le rapport entre l'énergie absorbée par la surface et l'énergie solaire incidente au voisinage de la normale. Le facteur d'émission total hémisphérique $\epsilon_{\Delta}$ est déterminé à partir de mesures d'absorption du rayonnement émis par un radiateur à $70^{\circ} \mathrm{C}$. Ce facteur représente le rapport entre l'énergie émise par la surface et l'énergie émise par un corps noir à la même température de $70^{\circ} \mathrm{C}$.

2.5 ANALYSE DES COMPosés ChIMIQUES. - Les profils de répartition des éléments dans les couches ont été obtenus par spectroscopie de masse des ions secondaires (SIMS). La zone analysée correspond à un cercle de $100 \mu \mathrm{m}$ de diamètre environ. La profondeur d'analyse est inférieure à $2 \mathrm{~nm}$. Les ions analysés sont : ${ }^{57} \mathrm{Fe}^{+},{ }^{53} \mathrm{Cr}^{+},{ }^{60} \mathrm{Ni}^{+},{ }^{34} \mathrm{~S}^{-}$et ${ }^{16} \mathrm{O}^{-}$.

La nature des composés cristallisés a été déterminée par diffraction des électrons rapides par réflexion. La technique et l'appareillage ont été décrits antérieurement [6,7]. L'aire analysée est de $200 \mu \mathrm{m}$ environ et l'on peut considérer que l'on travaille en pseudo-transmission, le pouvoir de pénétration du faisceau est dans le cas le plus favorable de $20 \mathrm{~nm}$.

\section{Caractéristiques des revêtements à l'état initial.}

3.1 PROPRIÉTÉS OPTIQUES. - Les valeurs des facteurs d'absorption et d'émission des revêtements sont indiquées sur la figure 2 en fonction du potentiel de traitement. La sélectivité spectrale des couches est excellente quelque soit le potentiel de traitement sauf au voisinage du potentiel de passivation $\left(E_{\mathrm{p}}\right)$.

En outre pour des potentiels voisins ou supérieurs à $E_{\mathrm{p}}$ la couche se détache du substrat métallique au cours de l'élaboration.

Il faut noter que des revêtements ayant une bonne sélectivité peuvent être obtenus à des potentiels sensiblement inférieurs au potentiel d'abandon. Dans ce domaine de potentiel le courant global est négatif parce que le courant d'oxydation est plus faible que le courant de réduction des espèces en solution. Des couches sélectives peuvent donc être obtenues par oxydation anodique dans le domaine de potentiel correspondant pratiquement à tout le domaine d'activité de l'acier [8]. Leurs propriétés optiques sont comparables à celles des revêtements élaborés par traitement chimique dans le même bain et à la même température [10]. La meilleure sélectivité est obtenue au voisinage du potentiel d'abandon (entre $-500 \mathrm{mV}$ et $-400 \mathrm{mV}$ ). Cela n'a rien de surprenant puisque pour pouvoir comparer les couches élaborées selon les 2 procédés, nous sommes convenus d'utiliser la même composition de bain et d'opérer à la même température ; ces conditions, correspondant aux conditions optimales pour le traitement par voie chimique, sont sensiblement reproduites au voisinage du potentiel d'abandon.

3.2 EPAISSEUR. - L'épaisseur des revêtements est appréciée à partir de la durée de décapage par bombardement ionique en admettant que la vitesse de décapage des différentes couches est sensiblement la même.

La figure 3 montre que les couches sélectives élaborées par traitement électrochimiques à des potentiels différents ont des épaisseurs pratiquement identiques. Cette épaisseur est la même que celle des couches élaborées par traitement chimique soit 150 à $160 \mathrm{~nm}$ [10].

\subsection{COMPOSITION.}

3.3.1 Analyse par spectroscopie de masse des ions secondaires. — Les profils des éléments (Fig. 3) 

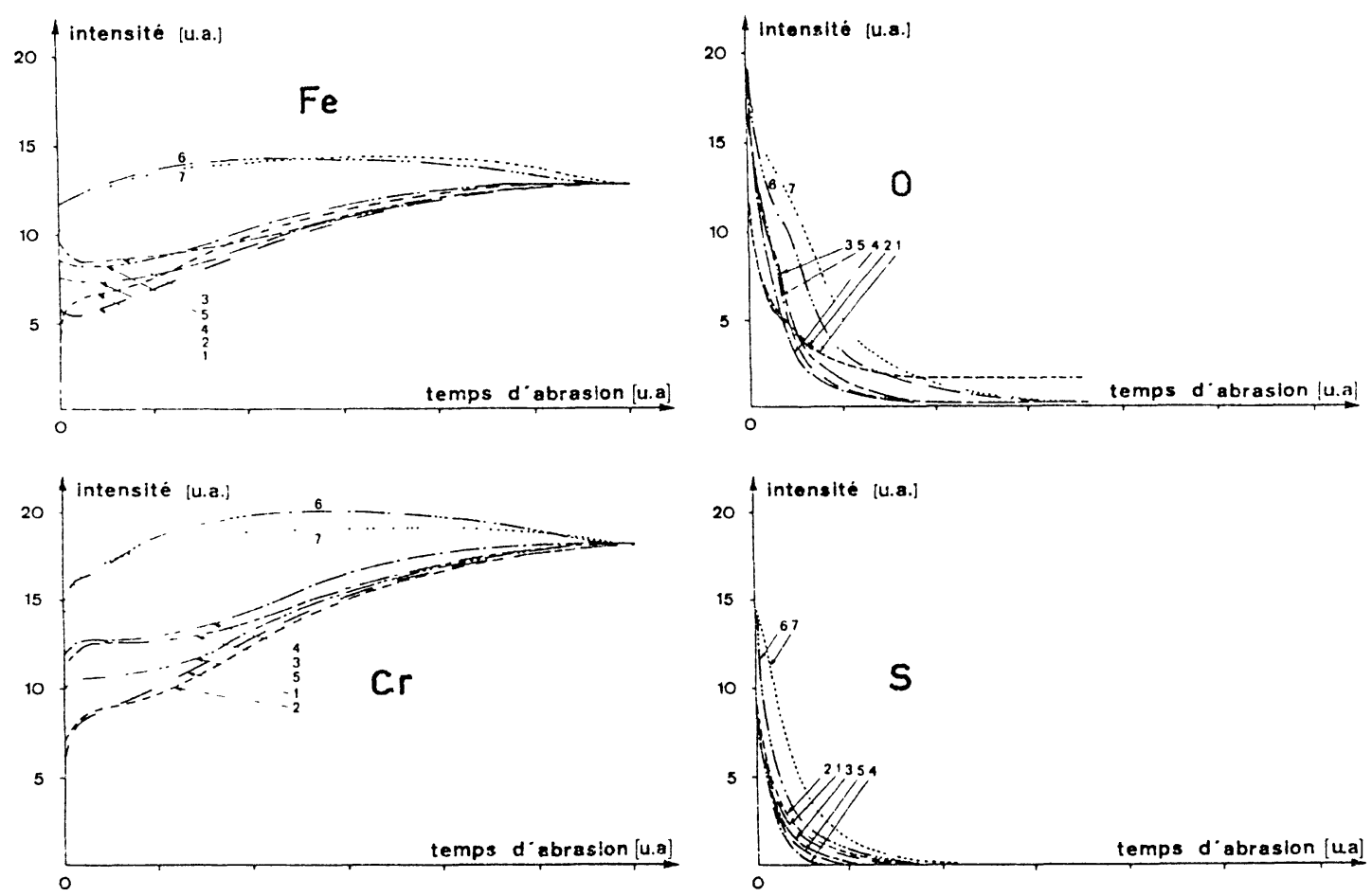

Fig. 3. - Influence du potentiel d'élaboration sur les profils de répartition des éléments dans la couche de conversion. 1) $-500 \mathrm{mV}$;2) $-480 \mathrm{mV}$;3) $-420 \mathrm{mV}$; 4) $-350 \mathrm{mV}$;5) $-300 \mathrm{mV}$; 6) $-250 \mathrm{mV}$; 7) $-200 \mathrm{mV}$. [Influence of preparation potential on SIMS profiles in the conversion coating.]

dans les différentes couches révèlent la présence d'un film superficiel et d'une zone profonde constituée de deux sous couches. Dans le film superficiel et dans la couche externe de la zone profonde les intensités ioniques de l'oxygène et du soufre sont très élevées tandis que celles du fer et du chrome y sont plus faibles que dans l'acier. Dans la couche interne de la zone profonde les concentrations de l'oxygène et du soufre tendent vers zéro tandis que celles du fer et du chrome tendent vers les valeurs correspondant au substrat.

Dans le cas du traitement par oxydation anodique l'allure des profils des éléments dépend de la valeur du potentiel appliqué (Fig. 2). Les intensités ioniques de l'oxygène et du soufre dans la couche et notamment dans la zone profonde, augmentent quand la valeur du potentiel croit et se rapproche de celle du potentiel de passivation. Cette augmentation pourrait traduire un accroissement de l'état d'oxydation du revêtement (degré d'oxydation des métaux plus élevé ou augmentation de la proportion de phase oxyde par rapport à la phase métallique). Les intensités ioniques du fer, du chrome et du nickel augmentent également quand le potentiel prend des valeurs plus élevées. En particulier, ces éléments sont fortement exaltés quand la valeur du potentiel appliqué est proche de celle du potentiel de passivation (Fig. 3, courbes 6 et 7). Cette exaltation des éléments métalliques pourait être en relation avec l'état d'oxydation du revêtement.

Les profils des éléments relatifs aux couches élaborées par un traitement anodique effectué à un potentiel voisin du potentiel d'abandon (Fig. 3, courbes 1 et 2) sont sensiblement les mêmes que celles obtenues par traitement chimique (6) (Fig. 4). 


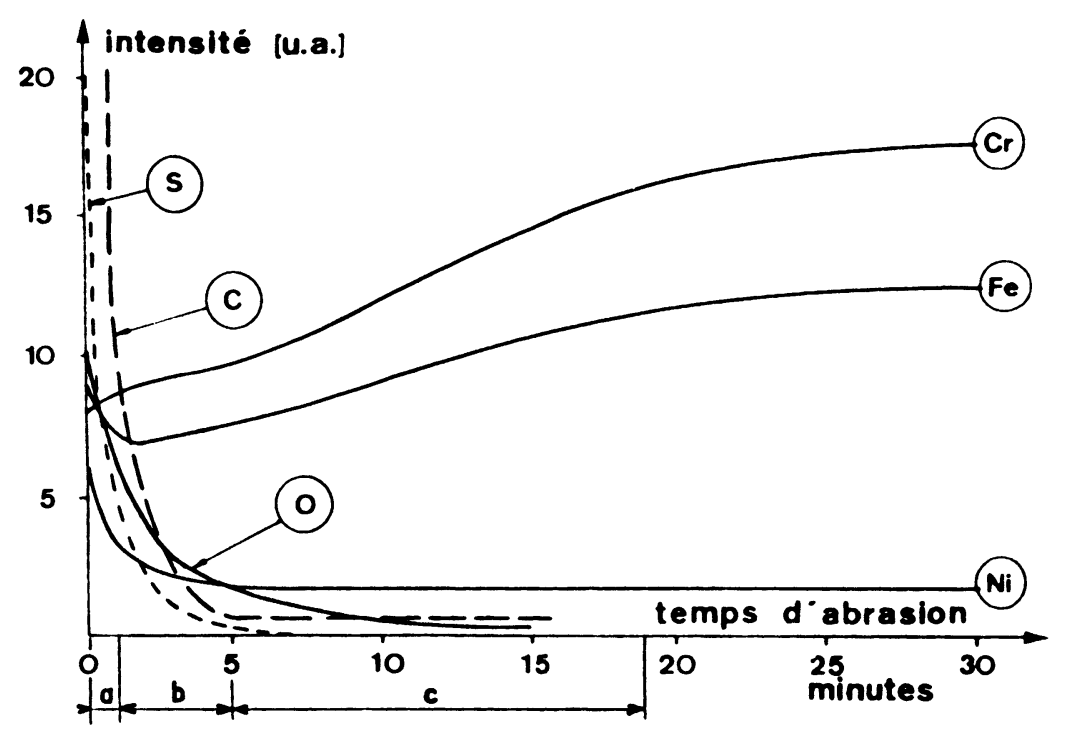

Fig. 4. - Profils de répartition des éléments dans la couche de conversion élaborée par traitement chimique. (a) Film superficiel, (b) couche externe et (c) couche interne de la zone profonde.

[Depth profile in the conversion coating prepared by chemical treatment. a) Superficial film. b) External zone (deep zone). c) Internal zone (deep zone).]

3.3.2 Analyse par diffraction électronique RHEED. - L'analyse RHEED des couches conduit à des diagrammes de même type : un dépouillement succinct montre qu'ils correspondent à la solution solide de ferrite et à de la magnétite substituée. Leur interprétation a donc été effectuée en comparant (Tabs. II, III, IV) les valeurs des distances interréticulaires et les intensités des raies observées aux calculs théoriques présentés par ailleurs [6].

Les raies observées du spinelle ont été attribuées à une magnétite faiblement substituée, à $5 \%$ de chrome au maximum. En outre l'observation de ces diagrammes figure 5, fait apparaitre l'influence du potentiel d'élaboration sur l'état de cristallisation du revêtement. Ainsi le diagramme obtenu à un potentiel de $-500 \mathrm{mV}$ est formé d'un nombre réduit d'anneaux et de halos noyés dans un fond continu intense ; l'aspect diffus des halos de magnétite montre que ce composé est présent sous forme de cristallites de taille inférieure à $10 \mathrm{~nm}$.

Les diagrammes obtenus à $-420 \mathrm{mV}$ et $-250 \mathrm{mV}$ présentent un fond continu de faible intensité, ils font apparaitre l'ensemble des raies élargies de la ferrite a sous forme d'anneaux fins et un nombre réduit de raies élargies de la magnétite. Ce qui indique que cette dernière est en faible proportion dans le revêtement.

La morphologie des couches qui apparait sur les micrographies de la figure 6 confirme ces observations.

Ces résultats sont à rapprocher des observations effectuées à partir de couches élaborées par voie chimique [6]. Celles-ci sont constituées pour leur partie cristallisée de ferrite et d'une faible proportion de magnétite faiblement substituée (5\% de chrome au maximum). L'état de cristallisation est tout à fait semblable à celui des revêtements obtenus à bas potentiel $(-500 \mathrm{mV})$. 
Tableau II. - Couche élaborée à $-500 \mathrm{mV}$.

[Coating prepared at $-500 \mathrm{mV}$.]

\begin{tabular}{|c|c|c|c|c|c|c|c|}
\hline \multirow{2}{*}{\multicolumn{2}{|c|}{$\begin{array}{l}\text { VALEURS } \\
\text { MESURÉES }\end{array}$}} & \multicolumn{6}{|c|}{ VALEURS CALCULÉES } \\
\hline & & \multicolumn{3}{|c|}{$\mathrm{Fe} \alpha$} & \multicolumn{3}{|c|}{$\mathrm{Fe}_{3} \mathrm{O}_{4}$} \\
\hline$I$ & $d_{h k l}$ & $h k l$ & $d_{h k l}$ & $I_{\text {cal }}$ & $h k l$ & $d_{h k l}$ & $I_{\text {cal }}$ \\
\hline \multirow[t]{2}{*}{ halo f } & 5,501 & & & & 111 & 4,847 & 304 \\
\hline & 2,851 & & & & 220 & 2,968 & 1248 \\
\hline \multirow[t]{2}{*}{$\mathrm{i}$} & 2,712 & & & & & & \\
\hline & 2,517 & & & & 311 & 2,531 & 4151 \\
\hline \multirow[t]{2}{*}{$\mathrm{mi}$} & 2,139 & & & & 400 & 2,099 & 1152 \\
\hline & 2,026 & 110 & 2,027 & 188 & & & \\
\hline \multirow[t]{2}{*}{ f } & 1,774 & & & & 511 & 1,615 & 908 \\
\hline & 1,604 & & & & & & \\
\hline \multirow[t]{2}{*}{$\mathrm{mi}$} & 1,510 & & & & 440 & 1,484 & 1786 \\
\hline & 1,437 & 200 & 1,433 & 22 & & & \\
\hline \multirow[t]{2}{*}{ f } & 1,184 & 211 & 1,170 & 36 & & & \\
\hline & 1,160 & & & & & & \\
\hline
\end{tabular}

\section{Stabilité thermique des revêtements.}

4.1 VARIATION DES PROPRIÉTÉS OPTIQUES EN FONCTION DE LA TEMPÉRATURE DE TRAITEMENT THERMIQUE. - La figure 7 présente l'évolution, en fonction de la température, de la morphologie d'une couche obtenue à un potentiel voisin du potentiel d'abandon. La micrographie (a) montre que la couche de conversion initiale recouvre uniformément le substrat. On ne distingue pas les joints de grains de la tôle d'acier initiale. Après traitement thermique cet aspect reste inchangé jusqu'à $400^{\circ} \mathrm{C}$ (micrographie b). $\mathrm{A} 500^{\circ} \mathrm{C}$ la surface de l'échantillon devient beaucoup plus rugueuse (micrographie c). Il apparait aussi des traces d'oxydation superficielle localisées révélant le sens du laminage de la tôle ainsi que des petits nodules pouvant correspondre au carbure de niobium. La surface des échantillons traités à $600^{\circ} \mathrm{C}$ (micrographie d) présente un fort relief et on observe une augmentation de la taille des grains. Après un maintien à $700^{\circ} \mathrm{C}$ on obtient une surface présentant un aspect assez homogène, mais le relief est très important et les joints de grains de l'acier de base apparaissent.

L'évolution des propriétés optiques avec la température, pour différents potentiels d'élaboration, est présentée figure 8.

Les couches obtenues par oxydation anodique à un potentiel peu élevé $(-500 \mathrm{ou}-420 \mathrm{mV})$ (Fig. 8, courbes 2 et 3 ) ont un comportement analogue à celui des couches élaborées par traitement chimique. La sélectivité est maintenue jusqu'a $400^{\circ} \mathrm{C}$ environ ; les propriétés optiques de ces couches restent inchangées jusqu'à $150^{\circ} \mathrm{C}$, au-delà on note la diminution du facteur d'émission qui conserve une valeur faible jusqu'à $450^{\circ} \mathrm{C}$ environ. Dans ce même domaine de température le 

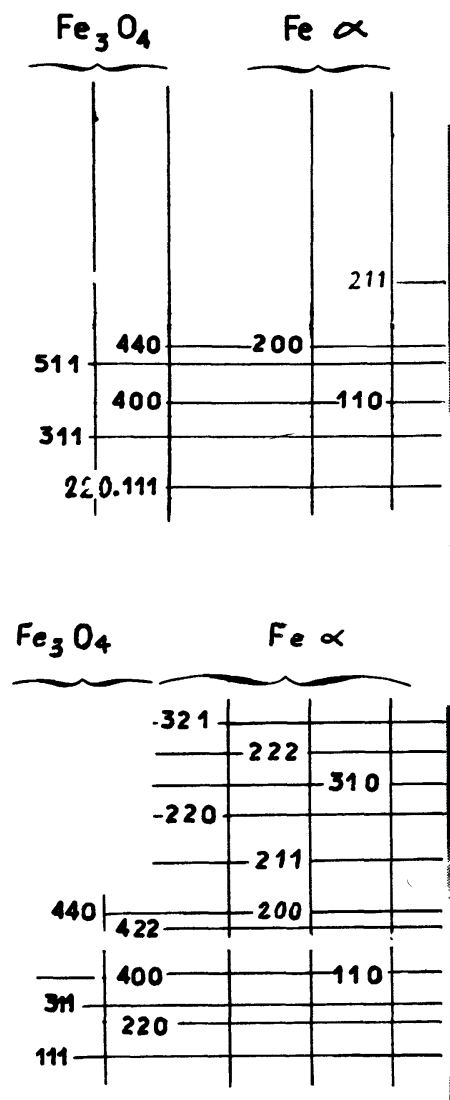

$-500 \mathrm{mV} / \mathrm{ecs}$
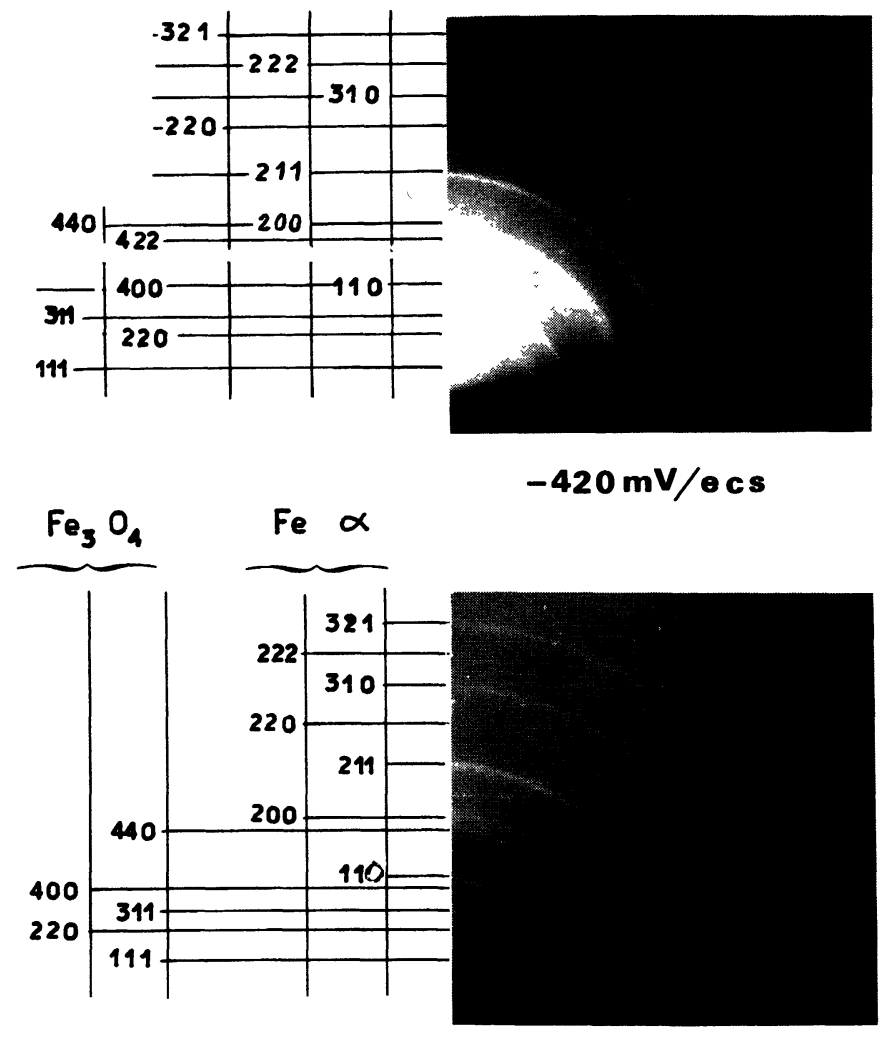

$-420 \mathrm{mV} / \mathrm{ecs}$

$$
-250 \mathrm{mV} / \mathrm{ecs}
$$

Fig. 5. - Diagrammes de diffraction de couches obtenues à différents potentiels d'élaboration. [Electron diffraction pattern of coating prepared at different potentials.] 
Tableau III. - Couche élaborée à $-420 \mathrm{mV}$.

[Coating prepared at $-420 \mathrm{mV}$.]

\begin{tabular}{|c|c|c|c|c|c|c|c|}
\hline \multirow{2}{*}{\multicolumn{2}{|c|}{$\begin{array}{l}\text { VALEURS } \\
\text { MESURÉES }\end{array}$}} & \multicolumn{6}{|c|}{ VALEURS CALCULEES } \\
\hline & & \multicolumn{3}{|c|}{$\mathrm{Fe} \alpha$} & \multicolumn{3}{|c|}{$\mathrm{Fe}_{3} \mathrm{O}_{4}$} \\
\hline$I$ & $d_{h k l}$ & $h k l$ & $d_{h k l}$ & $\overline{I_{\text {cal }}}$ & $h k l$ & $d_{h k l}$ & $I_{\text {cat }}$ \\
\hline $\begin{array}{c}\text { m } \\
\text { halo }\end{array}$ & $\begin{array}{l}5,134 \\
3,851\end{array}$ & & & & 111 & 4,847 & 304 \\
\hline $\begin{array}{l}\text { halo } \\
\text { diff }\end{array}$ & $\begin{array}{c}2,962 \\
2,75\end{array}$ & & & & 220 & 2,968 & 1248 \\
\hline $\mathrm{mi}$ & $\begin{array}{l}2,656 \\
2,452\end{array}$ & & & & 311 & 2,531 & 4251 \\
\hline f & $\begin{array}{l}2,081 \\
2,005\end{array}$ & 110 & 2,027 & 188 & 400 & 2,099 & 1152 \\
\hline $\mathbf{m}$ & 1,711 & & & & 422 & 1,714 & 300 \\
\hline halo & 1,604 & & & & 511 & 1.615 & 908 \\
\hline m & $\begin{array}{l}1,528 \\
1,492\end{array}$ & & & & 440) & 1,484 & 1796 \\
\hline $\mathrm{mi}$ & $\begin{array}{l}1,453 \\
1,426\end{array}$ & 200 & 1.433 & 22 & & & \\
\hline $\mathrm{i}$ & $\begin{array}{l}1,184 \\
1,160\end{array}$ & 211 & 1,170 & 36 & & & \\
\hline f & $\begin{array}{l}1,026 \\
1,013\end{array}$ & 220 & 1,013 & 9 & & & \\
\hline m & $\begin{array}{l}0,916 \\
0,901\end{array}$ & 310 & 0,906 & 11 & & & \\
\hline f & $\begin{array}{l}0,831 \\
0,819\end{array}$ & 222 & 0,827 & 2 & & & \\
\hline $\mathbf{m}$ & $\begin{array}{l}0,770 \\
0,758\end{array}$ & 321 & 0,766 & 9 & & & \\
\hline f & $\begin{array}{l}0,726 \\
0,713\end{array}$ & 400 & 0,717 & 1 & & & \\
\hline $\mathbf{m}$ & $\begin{array}{l}0,679 \\
0,666\end{array}$ & 411 & 0,675 & 4 & & & \\
\hline f & $\begin{array}{l}0,641 \\
0,631\end{array}$ & 420 & 0,641 & 2 & & & \\
\hline II & $\begin{array}{l}0,611 \\
0,601\end{array}$ & 332 & 0,611 & 1 & & & \\
\hline ก & 0,583 & 412 & 0,585 & 1 & & & \\
\hline f & 0,563 & 431 & 0,562 & 2 & & & \\
\hline
\end{tabular}


Tableau IV. - Couche élaborée à - $250 \mathrm{mV}$.

[Coating prepared at $-250 \mathrm{mV}$.]

\begin{tabular}{|c|c|c|c|c|c|c|c|}
\hline \multirow{2}{*}{\multicolumn{2}{|c|}{$\begin{array}{l}\text { VALEURS } \\
\text { MESURÉES }\end{array}$}} & \multicolumn{6}{|c|}{ VALEURS CALCULEEES } \\
\hline & & & Fe $\alpha$ & & & $\mathrm{Fe}_{3} \mathrm{O}_{4}$ & \\
\hline$I$ & $d_{h k l}$ & hkl & $d_{h k 1}$ & $I_{\mathrm{cal}}$ & hkl & $d_{h k l}$ & $I_{\text {cal }}$ \\
\hline f & $\begin{array}{l}5,13 \\
3,85\end{array}$ & & & & 111 & 4,847 & 304 \\
\hline f & $\begin{array}{l}3,08 \\
2,75\end{array}$ & & & & 220 & 2,968 & 1248 \\
\hline m & $\begin{array}{l}2,656 \\
2,484\end{array}$ & & & & 311 & 2,531 & 4151 \\
\hline f & $\begin{array}{l}2,265 \\
2,110\end{array}$ & & & & 400 & 2,099 & 1152 \\
\hline $\mathbf{i}$ & $\begin{array}{l}2,059 \\
1,974\end{array}$ & 110 & 2,027 & 188 & & & \\
\hline$\underset{\text { halo }}{\mathbf{m}}$ & $\begin{array}{l}1,674 \\
1,481\end{array}$ & & & & 440 & 1,484 & 1786 \\
\hline $\mathbf{m}$ & $\begin{array}{l}1,426 \\
1,390\end{array}$ & 200 & 1,433 & 22 & & & \\
\hline $\mathbf{i}$ & $\begin{array}{l}1,181 \\
1,153\end{array}$ & 211 & 1,170 & 36 & & & \\
\hline m & $\begin{array}{l}1,025 \\
0,994\end{array}$ & 220 & 1,013 & 9 & & & \\
\hline m & $\begin{array}{l}0,916 \\
0,886\end{array}$ & 310 & 1,906 & 11 & & & \\
\hline f & $\begin{array}{l}0,829 \\
0,812\end{array}$ & 222 & 0,827 & 2 & & & \\
\hline $\mathbf{m}$ & $\begin{array}{l}0,764 \\
0,749\end{array}$ & 321 & 0,766 & 9 & & & \\
\hline $\mathbf{f}$ & $\begin{array}{l}0,672 \\
0,660\end{array}$ & 411 & 0,675 & 4 & & & \\
\hline f & $\begin{array}{l}0,639 \\
0,622\end{array}$ & 420 & 0,641 & 2 & & & \\
\hline II & $\begin{array}{l}0,612 \\
0,602\end{array}$ & 332 & 0,611 & 1 & & & \\
\hline f & $\begin{array}{l}0,563 \\
0,546\end{array}$ & 431 & 0,562 & 2 & & & \\
\hline
\end{tabular}




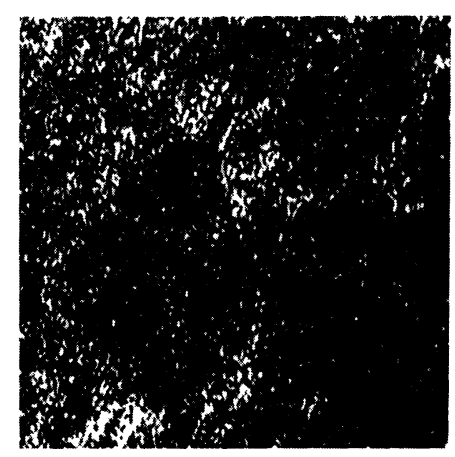

a) $10 \mu \mathrm{m}$

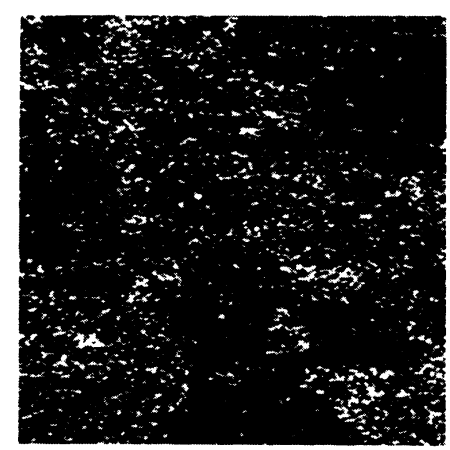

b) $10 \mu \mathrm{m}$

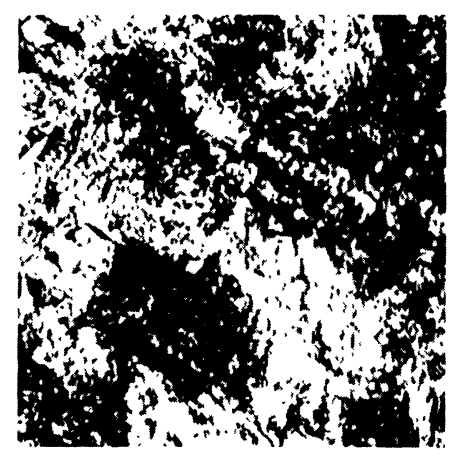

c) $10 \mu \mathrm{m}$

Fig. 6. - Evolution de la morphologie des surfaces sélectives en fonction du potentiel d'élaboration: a) couche élaborée à $-500 \mathrm{mV} / \mathrm{ECS}, \mathrm{b}$ ) couche élaborée à $-420 \mathrm{mV} / \mathrm{ECS}$, c) couche élaborée à $-250 \mathrm{mV} / \mathrm{ECS}$. [Variation of morphology of selective surfaces at different preparation potentials. a) Coating prepared at $-500 \mathrm{mV} / \mathrm{ECS}, \mathrm{b})$ Coating prepared at $-420 \mathrm{mV} / \mathrm{ECS}$, c) Coating prepared at $-250 \mathrm{mV} / \mathrm{ECS}$.] 


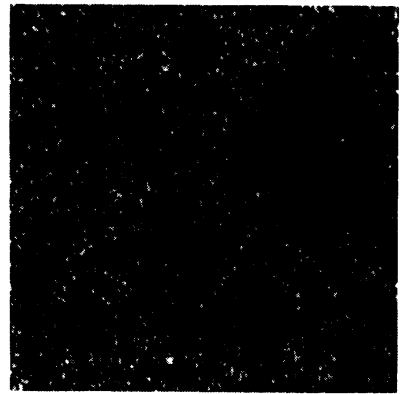

o) couche initiale $\left(a_{s}=0,96 . \quad \varepsilon_{20}=0,21\right)$

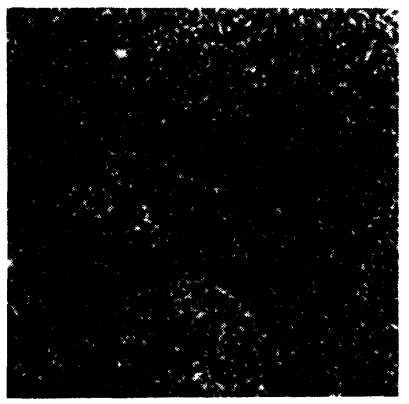

b) $400^{\circ} \mathrm{C}$ $l_{a_{s}}=0,94, \varepsilon_{20}=0,17$

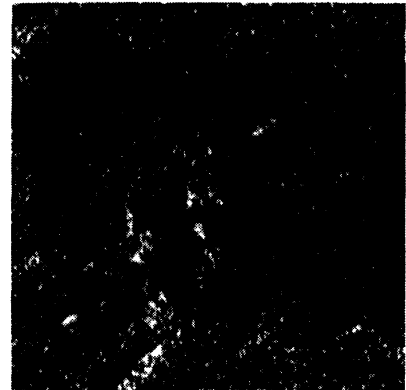

c) $500^{\circ} \mathrm{C}$ $\left(\alpha_{s}=0,89, \quad \varepsilon_{20}=0,185\right.$

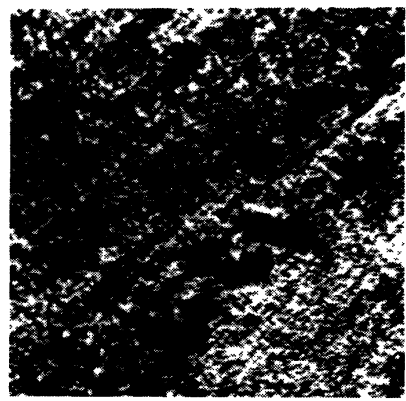

d) $600^{\circ} \mathrm{C}$ $\left(a_{s}=0,87, c_{20}=0,215\right)$

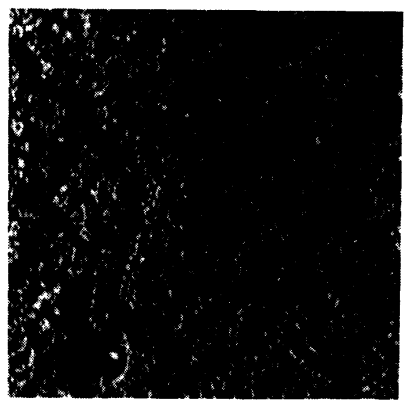

e) $700^{\circ} \mathrm{C}$ $l_{a_{5}}=0,82, c_{20}=0,31$

Fig. 7. - Evolution de la morphologie d'une couche pour différentes température du traitement thermique. [Variation of coating morphology with the temperature of heat treatment.] 
facteur d'absorption varie peu et conserve une valeur très élevée. A partir de $450^{\circ} \mathrm{C}$, on note une évolution continue de $a_{\mathrm{s}}$ et de $\epsilon_{\Delta}$ qui conduit à une diminution de la sélectivité des revêtements.

Pour les couches obtenues par oxydation anodique à potentiel élevé $(-250 \mathrm{mV})$ (Fig. 8, courbe 4) la sélectivité est détériorée dès $100^{\circ} \mathrm{C}$ à cause d'une forte diminution du facteur d'absorption jusqu'à des valeurs de l'ordre de 0,85 .

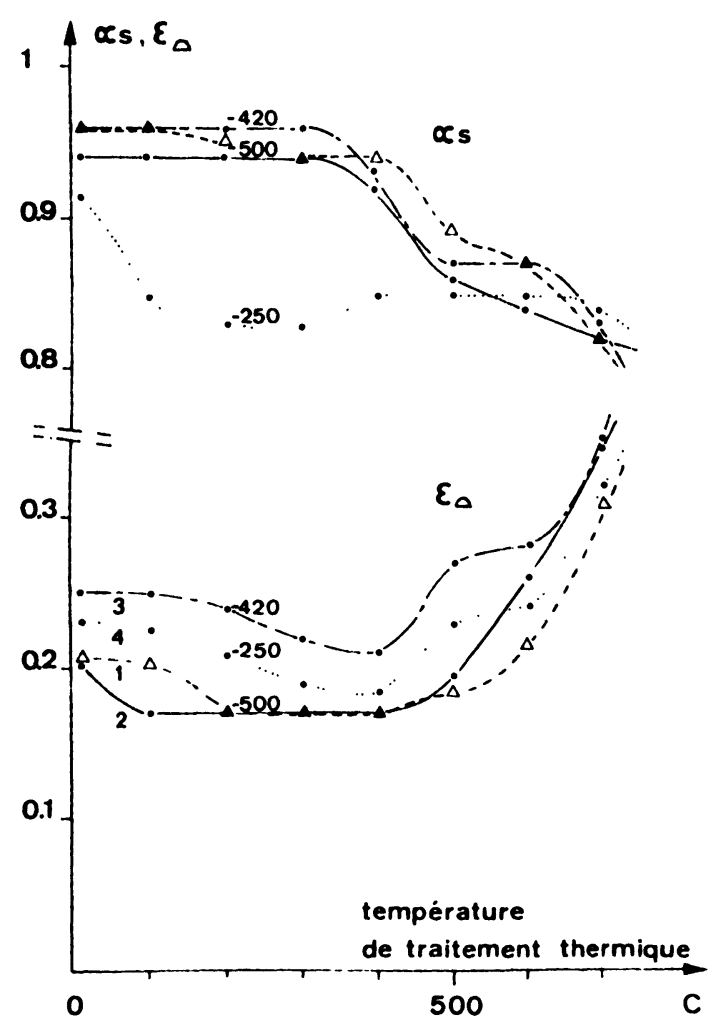

Fig. 8. - Variation des propriétés optiques en fonction de la température pour différents potentiels d'élaboration. 1) Traitement chimique, 2) $-500 \mathrm{mV}$. 3) $-420 \mathrm{mV}$. 4) $-250 \mathrm{mV}$.

[Variation of optical properties against temperature for different preparation potentials. 1) Chemical treatment. 2) $-500 \mathrm{mV} .3$ ) $-420 \mathrm{mV} .4$ ) $-250 \mathrm{mV}$.]

\subsection{VARIATION DE LA COMPOSITION DU MATÉRIAU EN COURS DE TRAITEMENT THERMIQUE.}

4.2.1 Analyse SIMS. - Les analyses SIMS montrent que le comportement à l'oxydation thermique des différentes couches est sensiblement le même dans le domaine des basses et moyennes températures (jusqu'à $400^{\circ} \mathrm{C}$ ) : l'épaisseur des couches varie peu et les profils des éléments métalliques sont peu modifiés après traitement thermique. Dans ce domaine de température l'oxygène diffuse progressivement ; cette diffusion est limitée à la seule couche de conversion. Il en est de même pour le soufre et sa teneur dans le revêtement tend à s'homogénéiser (Figs. 9, 10 et 11). 

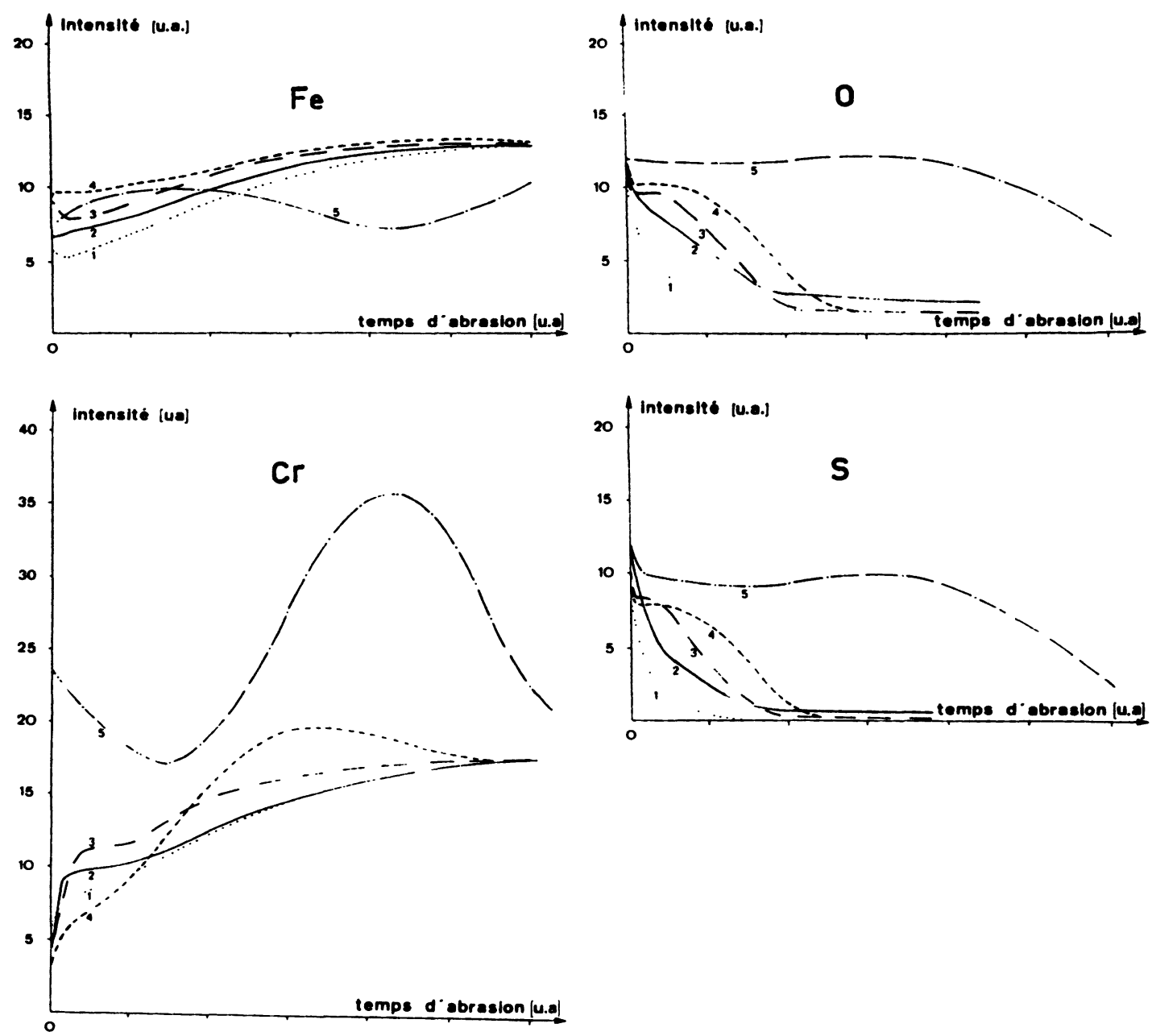

Fig. 9. - Evolution de profils de répartition des éléments en fonction de la température de traitement thermique. (potentiel d'elaboration : $-500 \mathrm{mV} / \mathrm{ECS}$ ). (1) état initial : (2) $200^{\circ} \mathrm{C}$, (3) $300^{\circ} \mathrm{C}$, (4) $400^{\circ} \mathrm{C}$, (5) $600^{\circ} \mathrm{C}$.

[Variation of SIMS profiles with heat treatment temperature (preparation potential : $-500 \mathrm{mV} / \mathrm{ECS}$ ).]

Au-delà de $450^{\circ} \mathrm{C}$, l'oxygène diffuse dans le substrat et l'épaisseur du revêtement augmente (courbes 5, Figs. 9, 10, 11) [9]. Le soufre diffuse également dans le substrat mais moins profondément que l'oxygène.

La diffusion progressive de l'oxygène révèle un phénomène d'oxydation de la couche qui commence dès $200^{\circ} \mathrm{C}$ puis du substrat à partir de $450^{\circ} \mathrm{C}$. Dans ce dernier domaine, on note que l'intensité ionique du chrome dans la couche et notamment à l'interface couche-substrat est fortement exaltée. Cette exaltation pourrait être due à la diffusion du chrome dans la couche depuis le substrat liée à l'oxydation de l'acier ou à celle des particules d'acier détachées ou non du substrat présentes principalement dans la partie interne de la zone profonde de la couche.

4.2.2 Analyse RHEED. - Nous avons étudié les couches élaborées par oxydation anodique à un potentiel peu élevé $(-500 \mathrm{mV},-420 \mathrm{mV})$; les couches obtenues à un potentiel voisin du 

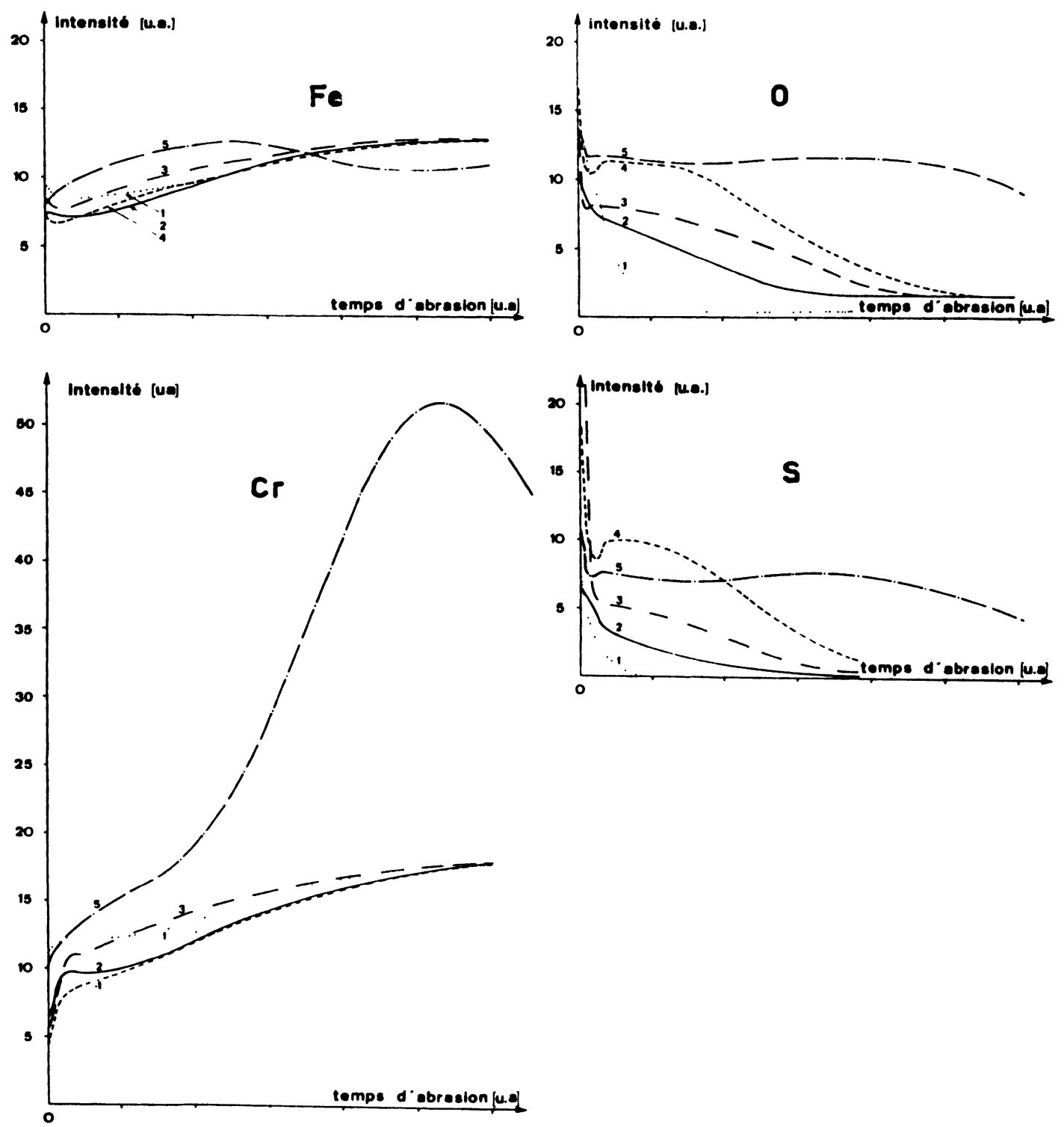

Fig. 10. - Evolution de profils de répartition des éléments en fonction de la température de traitement thermique. (potentiel d'élaboration : $-420 \mathrm{mV} / \mathrm{ECS}$ ). (1) $100^{\circ} \mathrm{C}$. (2) $200^{\circ} \mathrm{C}$. (3) $300^{\circ} \mathrm{C}$. (4) $400^{\circ} \mathrm{C}$. (5) $600^{\circ} \mathrm{C}$.

[Variation of SIMS profiles in function of heat treatment temperature (preparation potential : $-420 \mathrm{mV} / \mathrm{ECS})$.]

potentiel de passivation $(-250 \mathrm{mV})$ présentent moins d'intérêt puisque leur sélectivité se dégrade dès $100^{\circ} \mathrm{C}(11)$ [12]. Leur évolution en cours de traitement thermique est absolument analogue à celle obtenue par voie chimique (6), nous la présentons figure 12.

Jusqu'à $200^{\circ} \mathrm{C}$ la diffraction électronique ne permet pas de déceler de modifications de structure ou de composition. A partir de $200^{\circ} \mathrm{C}$ un nouveau type de diagramme apparaît. La confronta- 

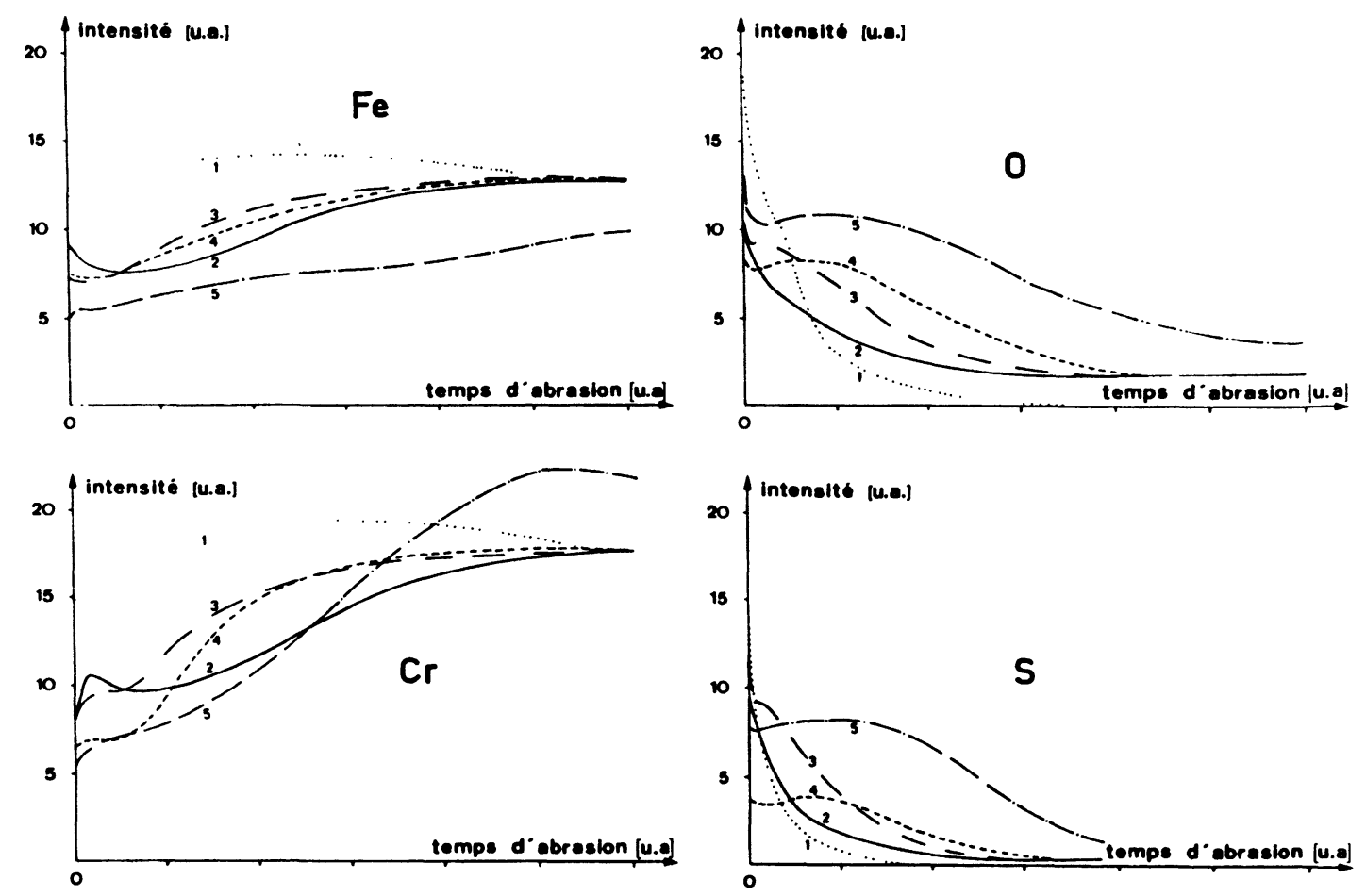

Fig. 11. - Evolution de profils de répartition des éléments en fonction de la température de traitement thermique. (potentiel d'elaboration : $-250 \mathrm{mV} / \mathrm{ECS}$ )

[Variation of SIMS profiles in function of heat treatment temperature (preparation potential : $-250 \mathrm{mV} / \mathrm{ECS})$.]

tion des résultats expérimentaux et des résultats calculés pour différents composés susceptibles de se former dans ce domaine de température [6] nous permet de conclure qu'entre $200^{\circ} \mathrm{C}$ et $500^{\circ} \mathrm{C}$ les diagrammes observés correspondent à un mélange d'hématite pure ou faiblement substituée et d'une phase $\left(\mathrm{Fe}_{1-x} \mathrm{Cr}_{x}\right)_{2} \mathrm{O}_{3}$ substituée avec $x=0,25$. L'aspect diffus des anneaux et le fond continu intenses montrent que ces 2 phases sont au premier stade de cristallisation dans le cas des couches oxydées à $200^{\circ} \mathrm{C}$. Entre $300^{\circ} \mathrm{C}$ et $400^{\circ} \mathrm{C}$ la faible contribution du fond continu et la finesse des anneaux indiquent que les composés sont mieux cristallisés. La dimension des cristallites de magnétite est supérieure à $10 \mathrm{~nm}$. A $500^{\circ} \mathrm{C}$ la répartition inégale des intensités le long des anneaux indique une augmentation de la taille des cristallites. La dimension des particules des 2 phases serait supérieure à $40 \mathrm{~nm}$.

Pour des couches oxydées à des températures supérieures à $500^{\circ} \mathrm{C}$, l'hématite $\alpha \mathrm{Fe}_{2} \mathrm{O}_{3}$ commence à disparaître tand is qu'apparaît le chromite de type spinelle $\mathrm{Fe} \mathrm{Cr}_{2} \mathrm{O}_{4}$. $\mathrm{Ce}$ chromite devient le composé majoritaire des couches oxydées à $600^{\circ} \mathrm{C}$, il est alors parfaitement cristallisé, la taille des grains allant de 50 à $100 \mathrm{~nm}$. La phase $\left(\mathrm{Fe}_{1-x} \mathrm{Cr}_{x}\right)_{2} \mathrm{O}_{3}$ resterait présente dans les couches oxydées jusqu'à $600^{\circ} \mathrm{C}$.

\section{Discussion et conclusion.}

Les couches que nous avons élaboré par oxydation anodique à un potentiel correspondant au do- 


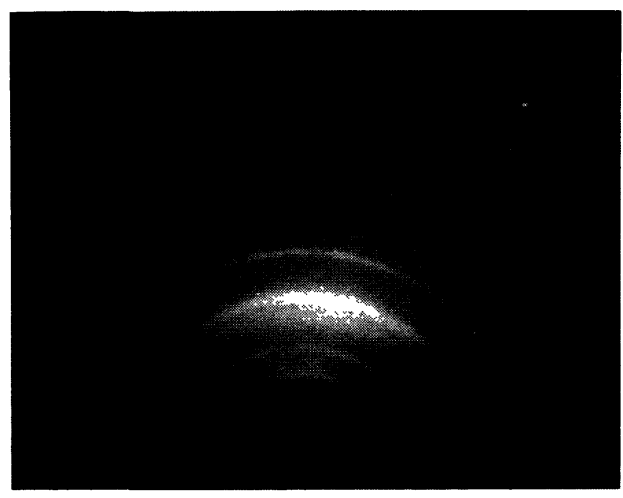

couche initiale

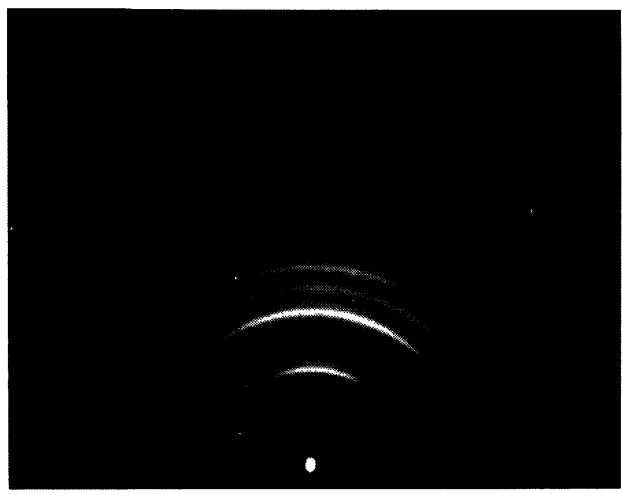

$300^{\circ} \mathrm{C}$

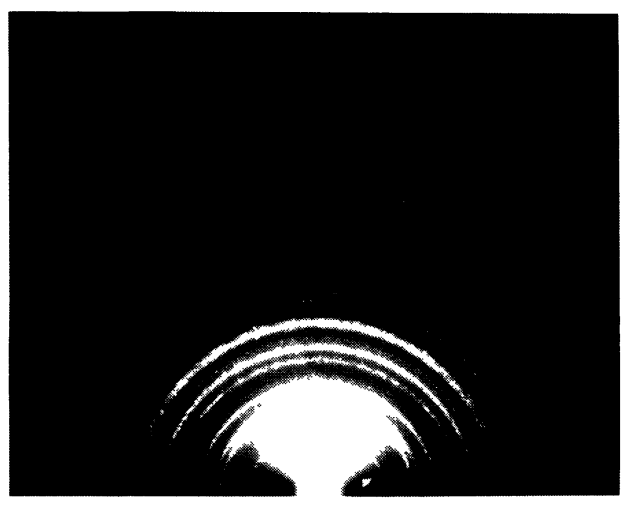

$600^{\circ} \mathrm{C}$

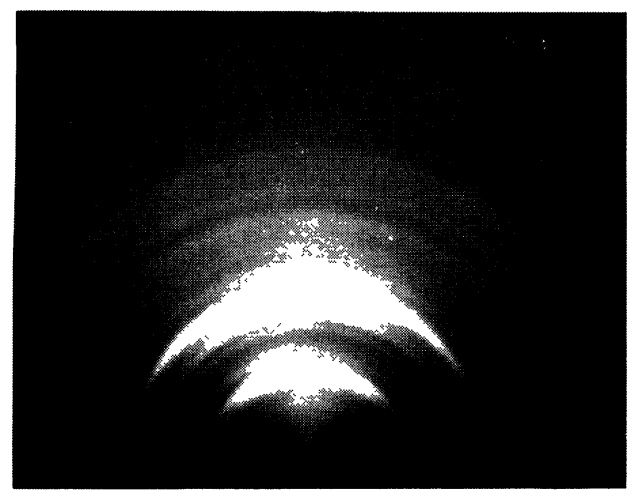

$200^{\circ} \mathrm{C}$

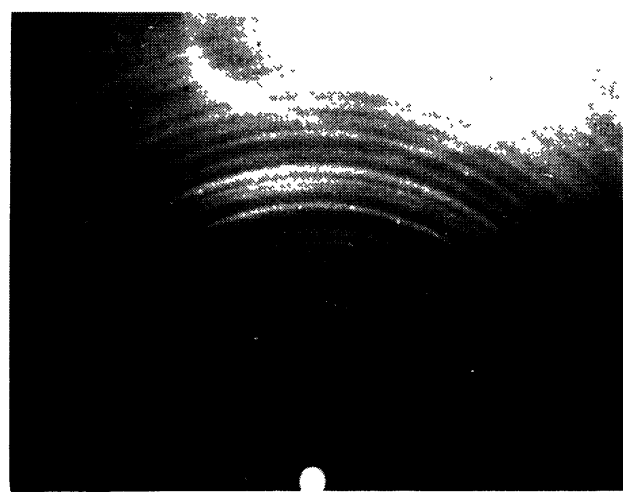

$500^{\circ} \mathrm{C}$

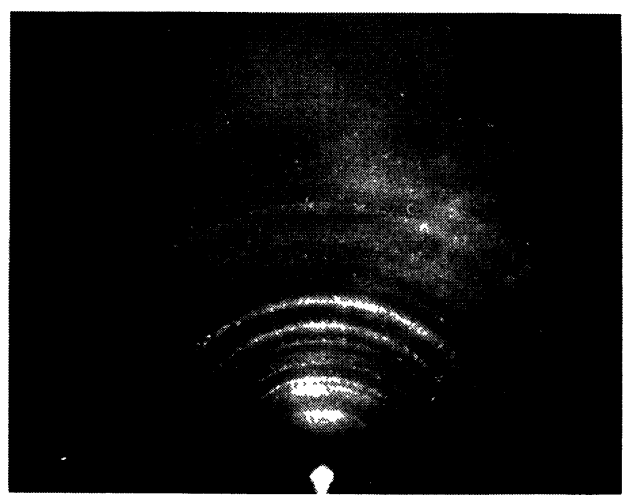

$700^{\circ} \mathrm{C}$

Fig. 12. - Diagramme de diffraction d'une couche élaborée à $-420 \mathrm{mV} / \mathrm{ECS}$ pour différentes températures de traitement thermique. L'indexation des diagrammes est identique à celle des diagrammes présentés dans la référence [6].

[Electron diffraction pattern of coating prepared at $-420 \mathrm{mV} / \mathrm{ECS}$ for different heat treatment temperatures.] 
maine d'activité de l'acier ont des propriétés optiques comparables à celles des couches obtenues par traitement chimique dans les mêmes conditions de température et de composition de bain. Les revêtements obtenus à un potentiel voisin du potentiel de passivation ont une sélectivité inférieure à celle des couches obtenues au voisinage du potentiel d'abandon $(I=0)$. Il est probable que les conditions expérimentales retenues correspondant aux conditions optimales de traitement par voie chimique conviennent d'autant mieux pour le traitement par électrolyse que le potentiel de travail est plus proche du potentiel d'abandon.

L'égalité d'épaisseur totale observée pour les couches que nous avons préparées tant par voie chimique que par voie électrolytique près du potentiel d'abandon justifie que nous ayons obtenu des revêtements ayant des propriétés optiques semblables. En effet l'épaisseur des couches est un facteur très important qui influence leurs propriétés optiques ; nous avons déjà montré les relations qui existent entre l'épaisseur de la couche et les facteurs d'absorption et d'émission [10].

Pour interpréter l'affaiblissement de la sélectivité des couches de même épaisseur élaborées près du potentiel de passivation, il faut faire appel à d'autres paramètres tels que : la composition chimique, l'état de cristallisation et la rugosité qui influencent $a_{\mathrm{s}}$ et $\epsilon_{\mathrm{o}}$. Pour leur partie cristallisée, la composition chimique des phases constitutives des couches élaborées par traitement électrochimique est sensiblement la même que celles obtenues par traitement chimique. Elles sont constituées de magnétite faiblement substituée (moins de $5 \%$ de chrome) et de particules d'acier détachées ou non du substrat pendant le traitement.

Cependant dans le cas du traitement électrolytique, les profils des éléments dépendent de la valeur du potentiel d'électrode. En particulier les teneurs en oxygène et en soufre de la zone profonde du revêtement augmente quand on élève la valeur du potentiel. Il en résulte un épaississement de la couche externe à teneur en oxygène élevée aux dépens de la couche interne dans laquelle prédomine la phase métallique. La figure 13 montre que l'augmentation d'épaisseur de la couche oxydée se produit pour des potentiels supérieurs à $-350 \mathrm{mV}$. L'épaisseur de cette couche (couche externe de la zone profonde) est de l'ordre de $35 \mathrm{~nm}$ au voisinage du potentiel d'abandon et de $110 \mathrm{~nm}$ environ pour un potentiel proche du potentiel de passivation. Pour ces dernières l'épaisseur de la couche interne est très faible (30 nm environ), alors qu'elle est de l'ordre de $100 \mathrm{~nm}$ pour les couches "chimiques". Il est intéressant de noter que dans un large domaine de potentiel, entre $-500 \mathrm{mV}$ et $-350 \mathrm{mV}$ l'épaisseur de la sous couche reste inchangée ; dans ce domaine de potentiel la réduction du proton qui conduit à un dégagement d'hydrogène sur l'électrode est thermodynamiquement possible.

Les couches élaborées par traitement électrochimique à des potentiels peu élevés (inférieurs à $-350 \mathrm{mV}$ environ) et celles obtenues par traitement chimique sont le siège d'un dégagement d'hydrogène. Ce dégagement pourrait ralentir la formation d'espèces oxydées dans la couche interne de la zone profonde ; en outre des réactions de réduction par l'hydrogène naissant de composés formés par oxydation du substrat n'est pas à exclure. Aux potentiels élevés la couche se développe en absence de dégagement d'hydrogène ; ces valeurs élevées du potentiel correspondent donc à des conditions plus favorable aux phénomènes d'oxydation. C'est à ce mécanisme d'oxydation que nous avons attribué l'augmentation d'épaisseur de la couche externe des revêtements élaborés à des potentiels supérieurs à $-350 \mathrm{mV}$.

Nous avons montré que pour des revêtements élaborés à des potentiels inférieurs ou égaux à $-350 \mathrm{mV}$, comme pour les couches élaborées par voie chimique nous obtenions des surfaces sélectives à stabilité thermique élevée $\left(T \leq 450^{\circ} \mathrm{C}\right)$. Cependant dans ce domaine de température, il apparaît des modifications importantes de composition. En particuliers la diffusion progressive de l'oxygène entraine un phénomène d'oxydation de la couche et notamment d'oxydation de la magnétite qui conduit à la formation d'hématite [13-15]. Les propriétés optiques de l'hématite sont moins favorables que celles de la magnétite par rapport à l'obtention d'une sélectivité spectrale élévée (le facteur d'absorption spectral dans le visible est de l'ordre de $10^{5} \mathrm{~cm}^{-1}$ pour 


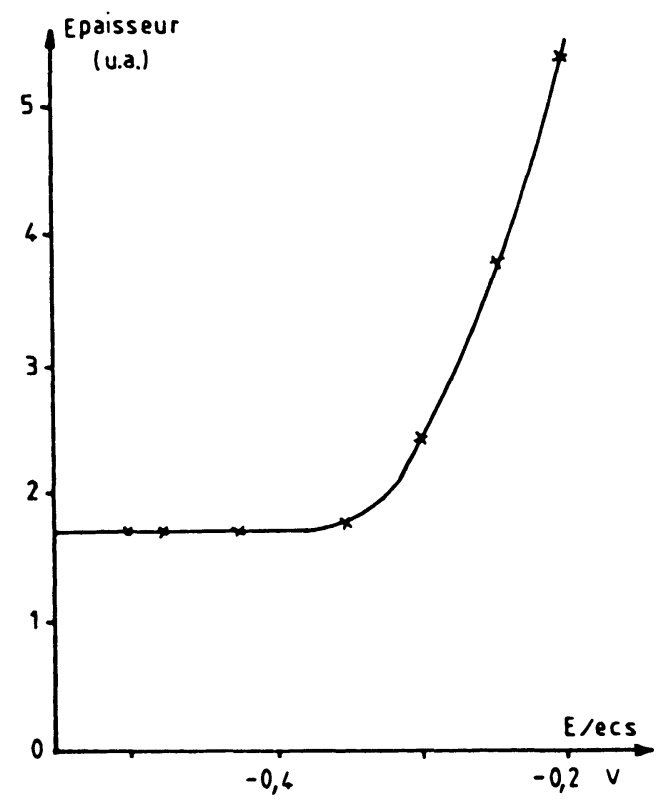

Fig. 13. - Evolution de l'épaisseur de la couche externe (partie b, Fig. 4) de la zone profonde en fonction du potentiel d'élaboration.

[Variations of depth of external zone (part b Fig. 4) of deep zone against preparation potential.]

$\mathrm{Fe}_{3} \mathrm{O}_{4}$ et de $10^{4} \mathrm{~cm}^{-1}$ pour l'hématite). Il est raisonnable de penser que la sélectivité des couches est conservée jusqu'à $400^{\circ} \mathrm{C}$ ou $450^{\circ} \mathrm{C}$ parce que leur épaisseur est suffisamment élevée pour que le modèle initial n'en soit pas fondamentalement modifié : le revêtement peut toujours être considéré comme un mélange de phases métalliques et semi-conductrices, ces dernières étant entourées d'une couche relativement mince de phase plus isolante de type hématite.

Au delà de $400^{\circ} \mathrm{Cl}$ 'oxydation de substrat métallique provoque une augmentation de l'épaisseur des couches ; à partir de $500^{\circ} \mathrm{C}$, l'hématite commence à disparaître tandis qu'apparaît le chromite $\mathrm{Fe} \mathrm{Cr}_{2} \mathrm{O}_{4}$. Ces modifications chimiques et structurales très importantes entrainent progressivement la perte de la sélectivité.

En conclusion les surfaces sélectives, élaborées par traitement chimique et oxydation anodique ont sensiblement même épaisseur et, pour leur partie cristallisée, même composition. La vitesse de formation des couches obtenues par voie électrochimique augmente avec le potentiel appliqué dans un rapport de 1 à 10 .

Nous avons mis en évidence l'influence du potentiel par la variation d'épaisseur de la sous couche oxydée (partie externe de la zone profonde) qui s'étend au détriment de la couche interne à partir d'un potentiel de l'ordre de $-350 \mathrm{mV} / \mathrm{ECS}$ et jusqu'au potentiel de passivation.

La stabilité thermique des couches est préservée tant que l'on opère à des potentiels inférieurs à cette valeur critique de $-350 \mathrm{mV} / \mathrm{ECS}$. 


\section{Bibliographie}

[1] ARIÈs L., TRAVERSE J.P., Procédé de fabrication d'un absorbeur sélectif de capteur solaire et absorbeur sélectif obtenu, Brevet FR.ANVAR 7918 414, U.S. Patent nº 4405414 (1979).

[2] ARIÈs L., TRAVERSE J.P., Procédé de fabrication d'un absorbeur sélectif de capteur solaire et absorbeur sélectif obtenu, Brevet FR.ANVAR 81 13 815, U.S. Patent n 4444600 (1981).

[3] ARIÈs L., BONINO J.P., BENAVENTE R., TRAVERSE J.P., Absorbeurs sélectifs de l'énergie solaire élaborés à partir d'acier inoxydable : influence de la composition chimique et de l'état métallurgique de l'alliage sur les propriétés optiques, Mater. Res. Bull. 18 (1983) 781.

[4] ARIES L., BAZIARD Y., FRAYSSE D., TRAVERSE J.P., High performance selective surfaces from stainless steel for the photothermal conversion of solar energy, Solar Energy 36 (1986) 521-529.

[5] ARIÈs L., CALSOU R., FraYSSE D., TRAVERSE J.P., Growth of selective coating on stainless steel, Thin solid Films 151 (1987) 413.

[6] Ariès L., Calsou R., Flores J.A., Traverse J.P., Etude par diffraction des électrons rapides en réflexion de couches sélectives pour la conversion photothermique de l'énergie solaire, J. Microsc. Spectrosc. Electron. 14 (1989) 41.

[7] Bonnafous C., Butto C., Calsou R., Dubourg A., Pellicer A., Analysis 4 (1976) 79. LAFOURCADE L., de SAJA J.A., Diffraction des électrons. Société Française de Microscopie Electronique, Sept. 1973 (Paris 1975).

[8] FORT P., Thèse Université Paul sabatier, Toulouse (1990).

[9] ARIĖ L., El BAKKOURI M., ROY J., TRAVERSE J.P., CALSOU R., SEMPERE R., Thermal oxidation study of thin magnetite-based coating from ironchromium alloys Thin Solid Films 197 (1991) 143-155.

[10] ARIÈs L., FORT P., FLORĖS J.A., TRAVERSE J.P., Analysis of the conversion coating on ferritic stainless steel of selective absorbers. Solar Energy Mater. 14 (1986) 143.

[11] MCQUEEN H.J., SHAPIRO M.J., SHAPIRO M.M., FELDMAN D., Selection of materials for flat plate solar collectors J. Mater. Energy Sys ASM 2 (1980) 65.

[12] KARLSON T., VALKONEN E., Preparation, characterization and stability tests of selectively absorbing oxides on stainless steel Sol. Energy 34 (1985) 417.

[13] FeITKNECHT W., GallagheR K.J., Mechanisms for the oxidation of $\mathrm{Fe}_{3} \mathrm{O}_{4}$. Nature 228 (1970) 548.

[14] SIRONI G., Mechanism of low temperature oxidation of magnetites, Nature 219 (1968) 1036.

[15] VeYsSiÈres P., Ralier J., Garem H. et GrilhÉ J., Précipitation d'une phase de structure corindon à l'intérieur d'une matrice spinelle Philos. Mag. 40 (1979) 553. 